Die Krone der Heim'schen Werke ist die vierbändige „Geologie der Schweiz“, die „in erstaunlicher Zusammenfassung aller seiner und seiner Zeitgenossen Arbeit eine geologische Monographie seines Vaterlandes darstellt, die weit überragt, was andere Länder aufzuweisen haben, und zwar wegen der Vorzüge der Heim'schen Darstellungskunst in Wort und Zeichnung“ (ARBENz). Sie ist in ihrer Gesamtheit bis heute das große Nachschlagewerk geblieben.

Es darf nicht übersehen werden, daß auch in der fast unermeßlichen Flut von Aufsätzen zahlreiche Gesamtdarstellungen sich finden, die als geologische Portraits unvergänglich sind, wie „Die Mythen“, „Die Gipfelflur der Alpen“, „Die Geschichte des Zürichsees" usw. Aber seinem Arbeitstag war noch mehr aufgetragen. Auch die Luffarben, die Ballonfahrt, die Feuerbestattung, die Erziehung des Menschen beschäftigten ihn ernstlich und lange. Die Kynologie verdankt ihm ein grundlegendes Werk und nachhaltige Förderung. Der Tag und Nacht hilfsbereite, of vollkommen selbstlose Einsatz für geologische Gutachten würde allein schon genügend dramatische Spannung für eine Lebensbeschreibung geboten haben. Mit der gleichen Begeisterung setzt er sich für die Hasliwerke ein, wie er für die ungeschmälerte Erhaltung des Rheinfalles kämpft. Die Wahrheit war sein einziger Wegweiser durch Forschung und Leben.

\title{
PILIERS DE LA SCIENCE GÉOGRAPHIQUE GRECQUE
}

\author{
Pierre Crettaz et ERnst WinkLer
}

La géographie moderne de la Grèce n'est pas très riche en traités descriptifs, c'est pourquoi l'excellente étude du chef de Service d'Etudes au Ministère du Commerce de la Grèce, M. P. J. ILIOPOulos, est d'autant plus bienvenue*. Elle a pour objet * un foyer d'une civilisation si généreuse en œuvres géniales universellement reconnues ". Cet ouvrage sorti d'une thèse à la Sorbonne sous la direction du professeur ANDRÉ CHOLLEY représente un document géographique dans le sens progressif du mot.

Le poids repose sur l'analyse exacte et détaillée de l'ensemble du territoire, construite sur la description de la géographie physique : de la constitution, des terrains, du relief du sol, du climat et de l'hydrologie et de la flore de la région (d'une surface d'environ $2167 \mathrm{~km}^{2}$ ), et dont la résultante est la conception "d'un grand nombre de subrégions bien individualisées" - plaine d'Athène, de la Mésogée, de l'Eleusis, de Marathon, région de la Lauréotique et blocs montagneux Chacune de ces régions présente une vie économique particulière qui est décrite dans la seconde partie de l'ouvrage. L'auteur s'appuie sur le paysage agraire, dont les traits charactéristiques sont "l'étroitesse de la terre cultivable, les contrastes saisissants de l'économie rurale " et la grande variété des cultures avec prédominance des céréales, de la vigne, de l'olivier, des cultures maraichaires et de l'élevage.

A la suite ceux-ci sont situés par rapport à la situation géographique, leur exploitation économique, leur évolution et leur rendement dans les temps actuels, ce qui n'empêche pas l'auteur de nous projeter d'intéressantes images sur l'antiquité à ce sujet. La représentation charactéristique de certaines exploitations types, par exemple la ferme de la région de Marathon, de la vallée de Céphise, etc. constitue une valeur exceptionnelle. Les chapitres instructifs sur la colonisation, la population, le commerce se groupent autour d'un point central: la description du paysage d'Athène (la ville et Pirée). L'architecture occupe une place de premier ordre. L'industrie (malgré son origine lointaine - elle remonte à Platon - ) reste quelque peu à l'arrière-plan, ce qui en relation avec la Grèce entière se justifie. Mais l'auteur observe que l'Attique, grâce à sa situation et à sa population (la région compte à peu près $20 \%$ de la population totale) représente les $60 \%$ de la main d'œuvre industrielle et domine ainsi la vie économique du pays.

Il serait erroné de vouloir mentionner ici toute la richesse de documentation, tous les problèmes et impulsions méthodiques que soulèvent cet œuvre. Nous attirerons cependant pour terminer l'attention du lecteur sur ce livre qui est de première importance tant au point de vue régional que national et que nous croyons absolument digne des remarques de STRABO: "Tout y rappelle les Dieux qui l'ont choisie pour demeure et les héros qui furent les ancêtres des générations suivantes *. Nous souhaitons à la Grèce et à la science géographique que l'auteur qui a réussi de dépeindre un excellent portrait d'une région culturelle méditerranéenne puisse faire de même pour d'autres régions de sa patrie. Il a d'ailleurs poursuivi son œuvre par une excellente étude climatologique sur l'Egée; nous en reparlerons**.

*Iliopoulos, P. J.: L'Attique au point de vue physique et économique. Athène, 1951. P. Athanassiadis \& Cie. 376 pages, 36 figures, 64 photos.

** Iliopoulos, P. J.: Le climat de la région égéenne de la Grèce. Collection de l'Institut français d'Athènes. Dirigé par Octave MerLier. Athènes, 1952, 75 pages, 4 cartes, 7 figures. 
D'un caractère quelquepeu différent est l'œuvre monumentale soṛtie de la plume du géographe allemand A. Philippson***. Prévue en quatre volumes, le premier vient de paraitre, elle est le couronnement d'un travail fait avec patience et amour qui s'étend sur plusieurs décades. Aucun auteur étranger a consacré autant d'études à la géologie, la géographie physique et la géographie régionale que Philippson, c'est pourquoi une appréciation objective ne pourra être faite qu'en présence de l'œuvre complète. Néanmoins; ce premier volume démontre qu'il s'agit d'un ouvrage fondamental englobant l'ensemble des disciplines géographiques se rapportant à la Grèce.

Ce volume est caracterisé par une description minutieuse - critiquée à tort - de la topographie. Description topographique lato sensu du paysage naturel, physique et culturel, constituant l'essence de toute œuvre de géographie régionale réalisée, du moins dans ce premier volume, avec une rare maitrise. Il ne se borne pas à cette notion fondamentale, mais en démontre, dans la mesure du possible et ceci est certainement difficile si l'on pense au nombre restreint de recherches géographiques que possède la Grèce, son origine génétique. Nous devons ainsi à PHILIPPson d'instructives pages sur la région de Thessalie, de la cuvette du Spercheios, de la Grèce occidentale avec Euböa et sa région principale : l'Attique. Le paysage de la ville d'Athène est excellement dépeint tant au point de vue géographique qu'historique. La géographie économique n'y tient qu'une place secondaire, c'est pourquoi l'ouvrage susmentionné de M. ILIopoulos comble avantageusement cette lacune.

La situation décrite dans l'ensemble par PhiLippson est celle de 1930, elle possède donc un caractère historico-géographique et est un document d'histoire culturelle du paysage. Le complément de E. KIRSTEN ne fait qu'augmenter cette valeur en ne modifiant en rien la ligne directrice de l'ouvrage. Celui-ci représente le fondement d'une géographie moderne de la Grèce. Espérons que l'auteur voie l'achèvement de son œuvre et qu'ainsi nous ayons l'occasion de la commenter plus longuement.

Ces deux ouvrages d'une teneur scientifique exemplaire réjouiront le monde scientifique et les amis de la Grèce. Ils seront certainement une impulsion nouvelle pour d'autres recherches au pays de l'origine de l'humanisme.

\section{NEUIGKEITEN - NOVA}

Zur Siedlungsgeographie der Schweiz. Die mit dem Bevölkerungszensus der Schweiz von 1950 verbundene Wohnungszählung bietet interessante Hinweise aụf die Siedlungsstruktur des Landes, von denen im Folgenden einige wiedergegeben sind. Zunächst sei festgehalten, daß 19411950 die Zahl der bewohnten Gebäude um 68611 auf 672883 (11,4\%), die der Haushaltungen und Wohnungen um 165175 auf $1312204(14,4 \%)$ zugenommen hat, daß also der Wohnhauscharakter (vorwiegend 1-2-Familienhäuser) relativ konstant blieb. Die Behausungsziffer ist mit 7,0 etwas geringer als $1941(7,1)$, die bewohnten Gebäude haben also zahlenmäßig etwas rascher zugenommen als die Bevölkerung; es ist im ganzen somit eine, wenn auch geringe Auflockerung der Bautweise eingretreten, was auch die Baustatistik des BIGA bestätigt: vom Zuwachs der 44000 Gebäude mit Wohnungen in Gemeinden mit mehr als 2000 Einwohnern entfallen nicht weniger als 28000 auf Einfamilienhäuser. Auch seit 1860, als 347184 bewohnte Gebäude und eine mittlere Behausungsziffer von 7,2 ermittelt wurden, ist die Schweiz $(1910$, z. Z. der ausgesprochenen Verstädterung und des Mietskasernenbaues betrug die Behausungsziffer 8,0) ein Land offener und demnach im ganzen hygienischer Bauweise geblieben. Naturgemäß bestehen hierin regionale Differenzierungen, doch sind sie keineswegs so stark, daß wesentliche Qualifizierungen möglich wären: unter den Kantonen hatten (1950) die höchsten Behausungsziffern Genf (11,3), Basel-Stadt $(9,2)$, Luzern $(8,7), Z$ ug $(8,3)$ und Zürich (8,2), unter den Städten Genf (20,7), Lausanne (17,0), Luzern $(13,8)$, Freiburg $(13,6)$,.Lugano $(13,1)$, Vevey $(12,6)$ und Zürich $(12,4)$. Die geringsten kantonalen Ziffern verzeichneten Glarus (4,7), Appenzell A. Rh. (4,9), Appenzell I. Rh., Tessin (je 5,3) und Baselland, Aargau und Thurgau (je 5,8). Dennoch hat sich sowohl insgesamt als auch regional das Siedlungsbild der Schweiz im Lichte der Gebäude- und Wohnstatistiken in den vergangenen hundert Jahren erheblich verändert. Wohl finden sich noch mehr als drei Viertel der bewohnten Gebäude (511 171) in den Landgemeinden; in den Städten (Gemeinden über 10000 Ein vohnern) stehen nur deren 161712. Aber 1860 entfielen auf die Städte erst 36000 Gebäude, d. h. $10 \%$ des Gesamtbestandes, wogegen in den Gemeinden mit weniger als 1000 Einwohnern mehr als dreimal mehr gezählt wurden. Hinsichtlich der Struktur der Wohnungen mag noch interessieren, daß 1950 97,6\% aller Wohnungen Küchen hatten, wobei Uri mit 99,1\% an der Spitze stand. Die geringsten Werte verzeichneten Appenzell I. Rh. mit 94,1 und Obwalden mit 95,7\%, während die Städtekantone günstigere Zăhlen aufwiesen. Die 10000 ermittelten Leerwohnungen schließlich $(0,79 \%)$, lassen erkennen, daß offenbar, absolut gesehen, von einer Wohnungsnot kaum gesprochen werden. kann; doch dürften hier die regionalen Unterschiede (Städte) erheblich sein. Immerhin

*** Philippson, Alfred (und Kirsten, Ernst): Die griechischen Landschaften. Eine Landeskunde. Bd. I. Der Nordosten der griechischen Halbinsel. Frankfurt a. M. 1950/52. Vittorio Klostermann. 1087 Seiten, 12 Karten. 